\title{
Single-Stage Combined Surgery for Treating Neglected Bilateral Developmental Hip Dysplasia after Walking Age
}

\section{Abdul Latif Shahid, ${ }^{1}$ Islam Hussain, ${ }^{2}$ Farhad Alam, ${ }^{3}$ Muhammad Nazir Awan, ${ }^{4}$ Hana Khurshid, ${ }^{5}$ Zulqernain Butt ${ }^{6}$}

\begin{abstract}
Objectives: To determine outcome in Single Stage Surgery of Both Hips in Bilateral Developmental Dysplasia of Hip in Children after walking age.

Methods: This was a retrospective review of 20 hips in 10 patients ( 7 females and 3 males), operated at the children's hospital and the institute of child health, Lahore between 2014 and 2016. The age of patients was between 2 and 5 years. There were 10 hips in grade IV, 6 in grade III and 4 in grade II according to Tonnis classification. Both hips were operated in single stage. Open reduction of hip joint was done by anterolateral approach in all children. Salter osteotomy was done in every child while femoral shortening was needed in 10 hips. Outcome of single stage surgery was assessed by radiological assessment of Severin's scoring system and functional assessment of MacKay's scoring system at final followup after 2 years of suegery.

Results: There was no effect of age, gender, malnutrition, body mass index on outcome. According to Severin's scoring system, 14 hips $(70 \%)$ were in grade I while $6(30 \%)$ in grade II. Outcome was excellent in 6 hips (30\%), good in $13(60 \%)$ and fair in $1(5 \%)$ by applying MacKay's scoring system. Hip spica of one child was changed after one month due to wetting with urine. There was no hip dislocation or subluxation in any case.
\end{abstract}

Conclusion: Single stage surgery of both hips can be done safely in bilateral developmental dysplasia of hip by anterolateral approach in late presented children.

Key Words: Developmental dysplasia of hip, Single stage surgery.

How to Cite: Latif A.S, Hussain I, Alam F, Nazir M. A, Khurshid H., B. Zulqernain. Single-stage combined surgery for treating neglected bilateral developmental hip dysplasia after walking age. Esculapio.2020;16(04):70-73.

DOI: https://doi.org/10.51273/esc20.2516415

\section{Introduction}

$\mathrm{D}$ evelopmental dysplasia of the hip (DDH) is a broad term having many aspects depending on patient's age at diagnosis and can lead to no problem in future and sometimes permanent disability. ${ }^{1}$

The overall incidence worldwide ranges from 1 to 34 cases per 1000 births. $^{2}$ Hip screening of neonates by physical examination should be done for proper diagnosis. In younger children, ultrasonography is the best

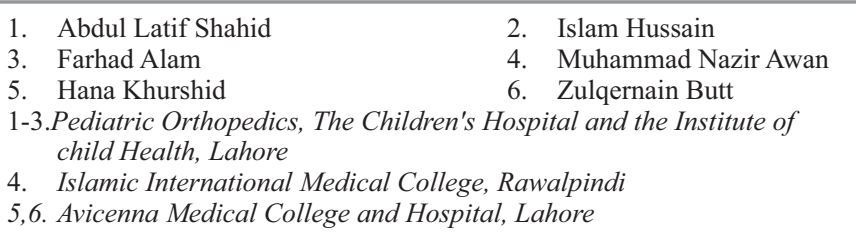

\section{Correspondence:}

Dr. Abdul Latif Shahid, Email: abdullatifshahid01@gmail.com

$\begin{array}{ll}\text { Submission Date: } & 20-08-2020 \\ \text { 1st Revision Date: } & 15-11-2020 \\ \text { Acceptance Date: } & 10-12-2020\end{array}$

while radiographs should be diagnostic modality in older children. ${ }^{2}$ The reason for late presentation of cases is delayed or missed diagnosis and failure in conservative or operative treatment. ${ }^{3}$ The incidence of late presentation is high in developing countries like Pakistan due to lack of awareness and negligence of family. Significant changes occur in joint with time such as dysplasia of the acetabulum, constriction of the acetabular capsule, fixation of the inversion of the limbus, thickened round ligament, infiltration of the acetabulum by fibrous tissue and femoral anteversion. If the problem is not treated in time, it will lead to early onset osteoarthritis and significant morbidities. ${ }^{4}$ Age of late presentation is different in different countries like after three months in Australia. ${ }^{5}$

Bracing, Traction, Closed reduction, Open reduction of hip with capsulorraphy and Pelvic or/and Femoral osteotomies are commonly used to treat DDH after 
walking age. ${ }^{6}$ The purpose of the DDH treatment is to achieve a stable hip joint by providing concentric reduction. This will stimulate normal development of femoral head and acetabulum., ${ }^{7,8}$ Children older than three years usually require open reduction combined with pelvic and femoral osteotomy. ${ }^{7}$ The main objective of treatment is to provide ideal environment of both femoral head and acetabulum for their development ${ }^{7}$. Most popular surgical technique to correct the deformity is combined open reduction with femoral shortening. ${ }^{9}$ Multiple studies have shown improved outcome in combination of open reduction, femoral shortening and pelvic osteotomies in single stage.' There is no conclusive evidence in the literature to support pre-treatment traction methods. ${ }^{8}$ The rationale of my study is to determine outcome of single stage surgery of both hips in bilateral developmental dysplasia of hip in children after walking age. The result of this study will allow us to change our previous concepts regarding management of bilateral developmental dysplasia of hip in children after walking age.

\section{Methods}

After approval from the ethical review committee of our hospital, this retrospective case series study was done. 10 patients with 20 hips who came in outpatient department of pediatric orthopedics of the Children Hospital and the Institute of Child Health, Lahore during 2014 and 2016 and fulfill the inclusion criteria were enrolled for this study. Our selection criteria were all patients aged 2-5 years with bilateral DDH. Children with age less than two years, who had taken initial treatment, paralytic, pathological, teratologic or traumatic dislocations and neuromuscular disease were excluded from the study. Informed consent and demographic profile (name, age, sex, and contact) was obtained. The procedure details, benefits and complications explained to parents. Preoperatively radiograph of pelvis including both hips anteroposterior view was taken and acetabular index was measured. Clinical data of all the patients like pain symptoms, range of hip joint motion, status of Trendelenburg sign and gait pattern were recorded for all the patients. The degree of dislocation of the femoral head was assessed by Tonnis classification system. A single surgical team carried out all procedures uniformly to reduce bias. Open reduction of both hip joints was done using anterolateral approach. Right side was operated first and then left side. Bilateral adductor tenotomy was done. Psoas tenotomy was done and intraarticular obstacles like pulvinar, ligamentum teres and transverse acetabular ligament were removed. Capsulotomy was done and femoral head was located within acetabulum. Decision about femoral shortening was made intraoperatively in case of difficulty in femoral head reduction. Salter osteotomy was performed if femoral head coverage was deficient. Capsulorrhaphy was done on both sides in all patients. After right side, left side was operated similarly. Bilateral hip spica was applied for a period of ten weeks. All data was collected on single proforma. Follow up period was 2 years. After ten weeks, hip spica and wires were removed under general anaesthesia. Union was assessed radiologically on every visit for 3-6months. Now full weight bearing was allowed and physiotherapy started assessing with radiological union. Dynamic compression plate used for femoral shortening was removed after one year under general anaesthesia. With evidence of occurrence of union on X-Ray, walking was advised. The outcome was assessed postoperatively by modified MacKay's criteria (table 1) and Severin's scoring system (table 2 ) at final follow up visit after 2 years.

\section{Results}

A total of 10 patients (20 hips) were operated for bilateral DDH. There were seven females $(70 \%)$ and $3(30 \%)$ males. $10(50 \%)$ hips were in grade IV, $6(30 \%)$ hips in grade III and $4(20 \%)$ hips in grade II according to Tönnis classification. Mean preoperative acetabular index angles were $38.6^{\circ}$ for right hips and $38.2^{\circ}$ for left hips. After surgery, mean acetabular index angles were $19.6^{\circ}$ for right hips and $20.2^{\circ}$ for left hips. According to Severin's scoring system, 14 hips $(70 \%)$ were in grade I while 6 hips $(30 \%)$ in grade II. No patients were seen with Severin's Grade III, IV and V. The clinical outcome was excellent in 6 hips $(30 \%)$, good in 13 hips $(65 \%)$ and fair in 1 hip (5\%) by applying MacKay's scoring system. Hip spica of one child had to be changed after one month due to wetting with urine. There was no hip dislocation or subluxation in any case.

\section{Discussion}

DDH is unilateral in $80 \%$ cases $(60 \%$ on left side while $20 \%$ on right side) and bilateral in $20 \%$ cases. $^{7}$ In unilateral cases, initial treatment is Pavlik harness, then closed reduction and hip spica if brace treatment 
fails. Open reduction of hip with capsulorrhaphy combined with femoral and pelvic osteotomies(triple procedure) is treatment of choice in children after walking age. ${ }^{10}$ The results of our study found $70 \%$ hips in grade I and 30\% in grade II of Severin scoring system. According to Mackay scoring 30\% hips were in excellent condition, $60 \%$ good and $5 \%$ in fair condition. In our study no patient required subsequent operation for redislocation and subluxation. There was $50 \%$ reduction in mean acetabular index angles postoperatively. Several research works have evaluated the functional and radiographic outcomes of surgical reconstruction for DDH. Mansoor et al found excellent and good radiological and clinical results in the one-stage procedure (open reduction and proximal femoral derotation osteotomy) in 15 patients with DDH. Comparable to our study results he found $66.6 \%$ in Grade I and $26.6 \%$ Grade II of Severin scores in his patients. The difference was seen in Mackay scoring where his results showed excellent condition of hips in $40 \%$ vs $30 \%$ in our study, good condition in $40 \%$ vs $60 \%$. ${ }^{11}$ Similar to our study Qadir I et al found single stage procedure of open reduction, femoral shortening and pelvic osteotomy for treatment of DDH in older children with good to excellent functional and radiological outcomes. The results of his retrospective analysis was $28.6 \%$ in excellent condition vs $30 \%$ in our results, $44(57.1 \%)$ in good condition vs $60 \%$ of our study results. The only difference was that they found $9(11.7 \%)$ hips in fair condition whereas we had 5\% hips that were in fair condition and also we did not find any hip in poor condition while they had $2(2.6 \%)$ in poor condition. ${ }^{9}$ Contrasting results with $90 \%$ success rate have been documented by various researchers. In a case series study by Saqib et al, variation from our results was concluded. $34.8 \%$ of patients achieved grade I (excellent) of McKay's classification and $39.1 \%$ grade II (good) in single stage triple procedure of open reduction, femoral and pelvic osteotomy. ${ }^{4}$ The disparity was also shown in Severin grading as they found $95.7 \%$ below grade III whereas none of the patients in our study were in grade III. This difference could be due to the follow up period of one year. Kashif et al studied the outcome of one stage correc-tion of developmental dysplasia of hip (DDH) in children older than three years of age. They reported 14\% hips were in Severin grade III, $21 \%$ in grade II and $50 \%$ in grade I. According to
Modified Mackay's scoring system clinical outcome of $50 \%$ hips were excellent (stable hips with pain free full range of motion, no limp and negative Trendelenburg sign), 32\% hips were good, 11\% hips were fair and $7 \%$ hips were poor. ${ }^{7}$ The difference in their results from our study could be due to the time of clinical evaluation postoperatively as we had followed up for 24 months in contrast to their 12 month evaluation. Hung NN and Duc HH compared Outcome between Salter Innominate Osteotomy and Zigzag Osteotomy Combined Fibular Allograft for Developmental Dysplasia of the Hip in Children. Their results with Salter Innominate Osteotomy according to Modified Mackay's scoring system were excellent hips in $73.9 \%$, good in $18.3 \%$, Fair in $5.3 \%$ and poor in $2.5 \%$. Radiologically $77.1 \%$ were in Severin grade I, $14.8 \%$ in grade II, $4.9 \%$ in grade III and $2.5 \%$ in grade IV. ${ }^{6}$ Their results were different than our study which could be due to different preoperative management techniques used.

Baki et al treated 32 hips (22 patients) with developmental dysplasia by a single-stage open reduction through Ferguson's medial approach and Pemberton acetabuloplasty. The clinical results were excellent in 30 hips $(93.8 \%)$ and good in two $(6.2 \%)$ as per Mackay scoring. Radiological assessment showed that 29 hips $(90.6 \%)$ were rated as class I and three hips $(9.4 \%)$ were class II by Severin classification ${ }^{12}$. This difference in results from our study could be due to difference in methodology of the operation as they operated via a medial approach. Hosny MM evaluate the clinical as well as the radio-graphic results of management of bilateral DDH cases with Salter and Dega osteotomy. The final clinical outcome results with Salter technique were excellent in $30 \%$ hips, good in $40 \%$, fair in $25 \%$ and poor in $10 \%{ }^{13}$ The advantage of choosing single stage operation was short hospital stay with no need of repeated immobilization and decreased joint stiffness. Also changes in the sacroiliac joint and pubic regions are limited in the single stage operation, and there is less damage to the pelvic inlet, birth canal, sciatic nerve and vessels. ${ }^{14}$ The limitations of the present study comprise its retrospective nature and the limited number of children. Also it only represents the experience of a single institution without a comparative study. Future studies can be done to compare different techniques and also with different institutions. 
Table 1: Modified McKay Criteria

\begin{tabular}{ll}
\hline Excellent & $\begin{array}{l}\text { Stable, painless hip, no limp, negative } \\
\text { Trendelenburg sign, and a full range of movement }\end{array}$ \\
\hline Good & $\begin{array}{l}\text { Stable, painless hip, slight limp, negative } \\
\text { Trendelenburg sign, and a slight decrease in range } \\
\text { of movement }\end{array}$ \\
Fair & $\begin{array}{l}\text { Stable, painless hip, limp, positive Trendelenburg } \\
\text { sign, and limitation of movement }\end{array}$ \\
Poor & $\begin{array}{l}\text { Unstable or painful hip, or both; positive } \\
\text { Trendelenburg sign }\end{array}$ \\
\hline
\end{tabular}

Table 2: Severin's Scoring System

\begin{tabular}{|c|c|}
\hline Grade I & Normal appearance \\
\hline Grade II & $\begin{array}{l}\text { Mild deformity of the femoral head and neck or } \\
\text { the acetabulum }\end{array}$ \\
\hline Grade III & $\begin{array}{l}\text { Moderate deformity of the femoral head and neck } \\
\text { or the acetabulum or both }\end{array}$ \\
\hline Grade IV & Subluxation of the femoral head \\
\hline Grade V & $\begin{array}{l}\text { Articulation of the femoral head with a false } \\
\text { acetabulum }\end{array}$ \\
\hline Grade VI & Redislocation \\
\hline
\end{tabular}

\section{Conclusion}

The results conclude that single stage surgery of both hips can be done safely in bilateral developmental dysplasia of hip by anterolateral approach in children after walking age.

\section{Conflict of Interest: $\quad$ None}

\section{References}

1. Wilkinson A.G., Wilkinson S. Neonatal hip dysplasia: a new perspective. Neo Reviwes. 2010; 11(7): e349- e362.Nguyen Ngoc Hung and Hoang Hai Duc. "Comparative Outcome between Salter Innominate Osteotomy and Zigzag Osteotomy Combined Fibular Allograft for Developmental Dysplasia of the Hip in Children". EC Orthopaedics 9.4 (2018): 187-210.

2. Al-Essa RS, Aljahdali FH, Alkhilaiwi RM, Philip W, Jawadi AH, Khoshhal KI. Diagnosis and treatment of developmental dysplasia of the hip: a current practice of paediatric orthopaedic surgeons. J Orthop Surg (Hong Kong) 2017;25 (2): 2309499017717197

3. Li H, Ye W, Xu L, Li L, Zhu W, Zheng Z. Sequential one-stage combined procedure for treating bilateral developmental hip dysplasia after walking age. J Int Med Res. 2019;47(7):2901-2909.

4. Saqib M, Salman M,Hayat S, Khan MA, Shakirulla. Developmental dysplasia of the hip in older children; prospects of functional and radiological outcome following a single stage triple procedure. Ayub Med Coll Abbottabad 2019;31(3):427-31.

5. Studer K, Williams N, Antoniou G, et al. Increase in late diagnosed developmental dysplasia of the hip in South Australia: risk factors, proposed solutions. Med J Aust 2016;204:240.

6. Hung NN and Duc HH. Comparative Outcome between Salter Innominate Osteotomy and Zigzag Osteotomy Combined Fibular Allograft for Developmental Dysplasia of the Hip in Children. EC Orthopaedics 2018; 9.4: 187-210.

7. Kashif S, Khan BA, Sana ullah, Ihsan ullah. Outcome of one Stage Surgical Correction of Developmental Dysplasia of Hip (DDH) in Children Older than three years of age. PJMHS 2016; 10(1): 258-261.

8. Çiçekli O and Doğan M. Evaluation of surgical outcome in advanced age patients with developmental hip dysplasia. International Journal of Surgery 2018;52:44-49.

9. Qadir I, Ahmad S, Zaman A, Khan CM, Ahmad S, Aziz A. One-stage Hip Reconstruction for Developmental Hip Dysplasia in Children over 8 Years of Age. Hip Pelvis 2018;30(4):260-268.

10. Agarwal A, Gupta N. Risk factors and diagnosis of developmental dysplasia of hip in children. J Clin Orthop Trauma. 2012;3(1):10-14.

11. Mansoor AK, Kraidi B, Al-Naser LM. Simultaneous versus two stages surgical treatment of developmental dislocation of the hip with excessive femoral anteversion in children under the age of three years. Journal of Ideas in Health 2018;1(2):34-41

12. Baki ME, Baki C, Bünyamin $\mathrm{HA}$ and Özcan M. Single-stage medial open reduction and Pemberton acetabuloplasty in developmental dysplasia of the hip

13. Hosny MM, Issa KH. Bilateral developmental dysplasia of the hip: setting an upper age limit for open reduction. Egypt Orthop J 2015;50:259-62.

14. Ezirmik N, Yildiz K. Advantages of Single-stage Surgical Treatment with Salter Innominate Osteotomy and Pemberton Pericapsular Osteotomy for Developmental Dysplasia of Both Hips. JIMR 2012; 40: $748-755$.

\section{Authors Contribution}

SLA: Manuscript Writing

NZ: Study Concept andDesign

HI, AF: Data Acquisition

AN: Statistical Analysis

AANM: Data Interpretation

KH: Manuscript Review 